\title{
New formulas in area
}

\author{
Ehab esmail ahmed \\ Bank el taslef Street, Tahta, Sohag, egypt \\ E-mail mathehab75@yahoo.com
}

\begin{abstract}
This formula develops old theories to facilitate the solution The use of geometric spaces is very important in our live. In ancient times it took many steps to resolve the area of the triangle, but in this research, it will be easy to solve the area of triangle with one side and .two angles only and this saves time, effort and thinking

In Old times, solving rhombus area took a number of difficult steps, but in this research it .will be easily solved.

Generally, rhombus area is calculated by half multiplied the lengths of the two diagonals, but in but in this research area is calculated in terms of one diagonal length and an angle only

Parallelogram area is equal to multiplication of the base length by the perpendicular, but the new method uses diagonal length and angles of the diagonal only

Area of the rectangle equal multiplication of length and width, Using the new method diagonal length and angles of the diagonal are used only

Uses

1- triangle area in a new method

2- parallelogram area a new method

3 - rectangle area in a new method

4 - rhombus area in a new method

5 - square area in a new method
\end{abstract}

\section{Keyword}

The area; Triangle; rhombus (diamond); Parallelogram; rectang 


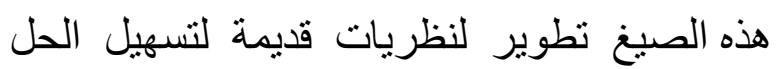

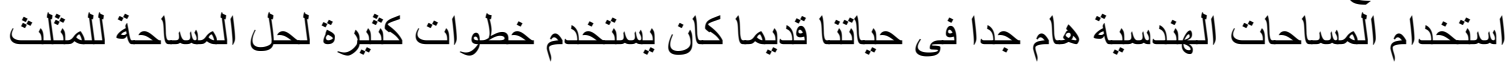

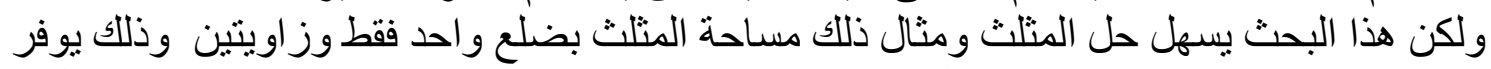

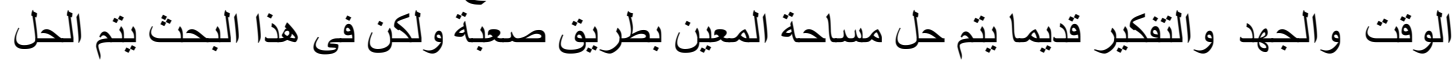

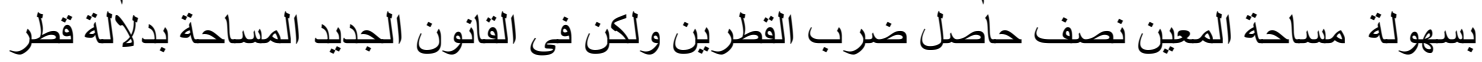

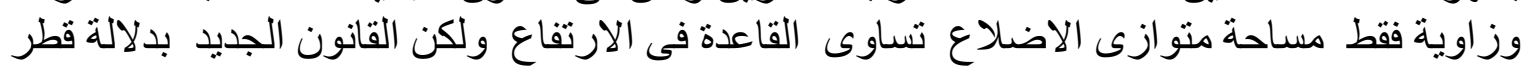

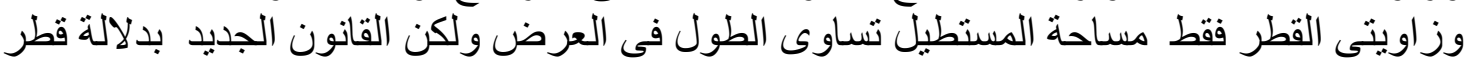

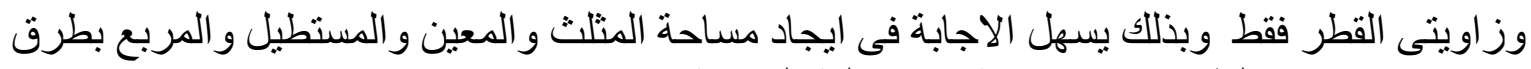
جديدة و هذا يفيد الطلاب فى هندسة مدنى كلية الهندسة 


\section{Introduction}

Calculating the area of a triangle using the length of one side and two angles is better than using the old solution solving rhombus area took a number of difficult steps, but in this research it will be easily solved

Generally, rhombus area is calculated by half multiplied the lengths of the two diagonals, but in but in this research area is calculated in terms of one diagonal length and an angle only

Parallelogram area is equal to multiplication of the base length by the perpendicular, but the new method uses diagonal length and angles of the diagonal only

Area of the rectangle equal multiplication of length and width, Using the new method diagonal length and angles of the diagonal are used only

\section{Idea of the research}

"The new methods"

The area of :
(1) Triangle
(2) rhombus (diamond)
(3) Parallelogram
(4) rectangle

\section{The area of triangle}

A

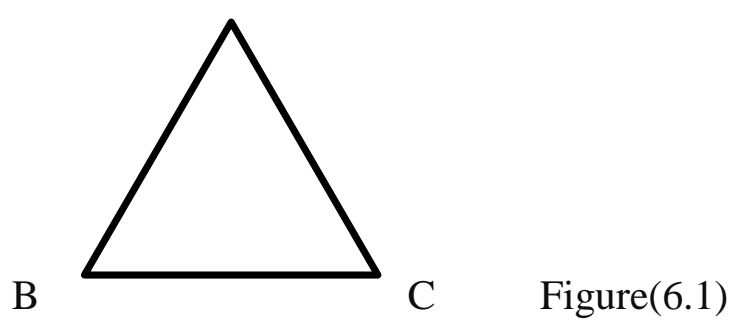


the area of the triangle

$=\frac{1}{2} \times(\mathrm{A} \mathrm{B}) \times(\mathrm{A} \mathrm{C}) \times \mathrm{SIN} \hat{\mathrm{A}}$

${ }_{0}^{0} 0 \frac{a c}{\operatorname{SIN} b}=\frac{a b}{\operatorname{SIN} c}$

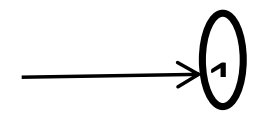

$0^{0} 0 \mathrm{AC}=(\mathrm{AB}) \times \mathrm{SIN}(\mathrm{B}) / \mathrm{SIN}(\mathrm{C})$

${ }_{0}^{0}{ }_{0}^{0} \operatorname{SIN~C}=\operatorname{SIN}(180-(\mathrm{A}+\mathrm{B}))$

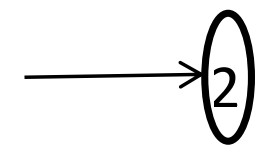

$$
=\operatorname{SIN}(\mathrm{A}+\mathrm{B})
$$

from (1), (2), (3)

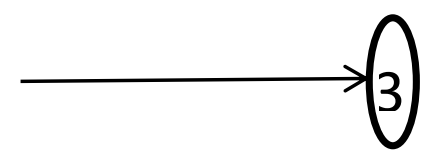

The area at triangle $=\frac{1}{2} \frac{(a b)^{2} \times \sin (a) \sin (b)_{0}}{\sin (a+b)} 0_{0}^{0}$

Example (1)

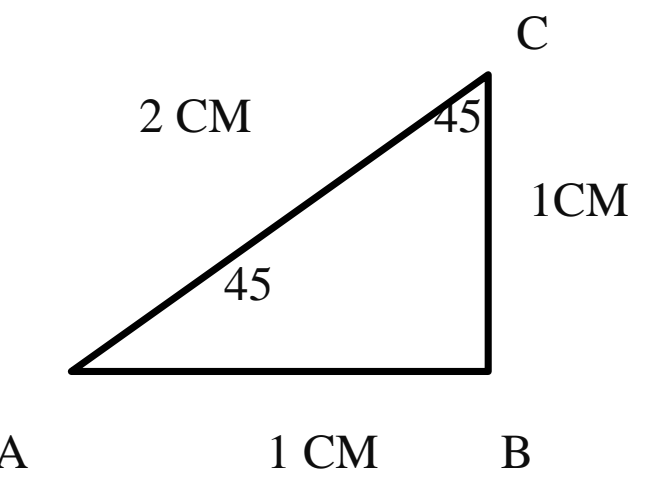

Solution (1)

The Area of triangle $a b c=\frac{1}{2} \mathrm{ab} \times \mathrm{b} \mathrm{c} \times \sin \mathrm{b}$

$$
=\frac{1}{2} \times 1 \times 1 \times 1=\frac{1}{2} \mathrm{~cm}^{2}
$$

Solution (2) by new method

The Area of triangle abc $=\frac{1}{2} \frac{(a b)^{2} \times \sin (a) \sin (b)}{\sin (a+b)}$

$=\frac{1}{2} \frac{(1)^{2} \times \sin (45) \sin (90)}{\sin (45+90)}$ 
$=\frac{1}{2} \times \frac{1 \times 1 \times \frac{1}{\sqrt{2}} \times 1}{\frac{1}{\sqrt{2}}}=\frac{1}{2} \mathrm{~cm}^{2}$

Example (1)

b

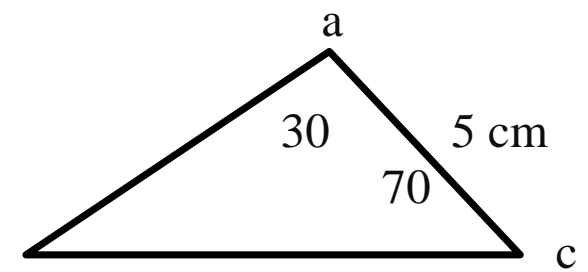

The Area oF $\triangle \mathrm{abc}=\frac{\frac{1}{2} \times(5)^{2} \times \sin (a) \times \sin (c)}{\sin (a+c)}$

$$
=\frac{\frac{1}{2} \times 25 \times \sin (30) \times \sin (70)}{\sin (100)}=5.96 \mathrm{~cm}^{2}
$$

\section{The area of parallelogram}

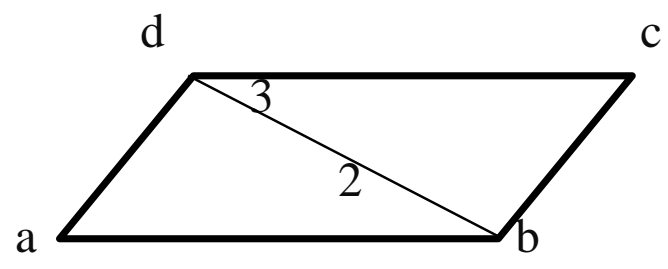

Figure (6.2)

\section{The proof}

The area of parallelogram a b c d

$$
=2 \times \text { area } \Delta \text { bd }
$$

$=z \times \frac{1}{z} \frac{(d b)^{2} \times \sin (3) \sin (2)}{\sin (2+3)}$
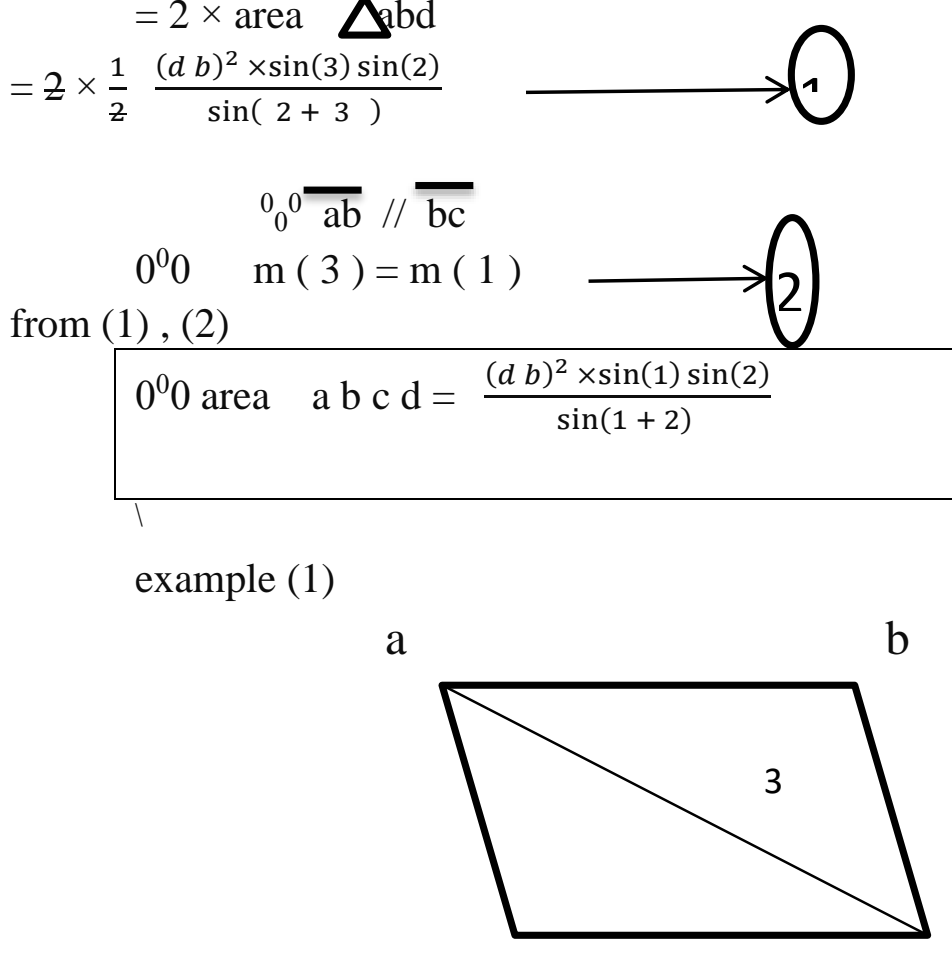
$5 \mathrm{~cm}$

$40 \quad 30$

d

c

Area of $\square$ a b c d $=\frac{(5)^{2} \times \sin (30) \sin (40)}{\sin (70)}$

$=8.55 \mathrm{~cm}^{2}$

The area of rhombus (diamond) 


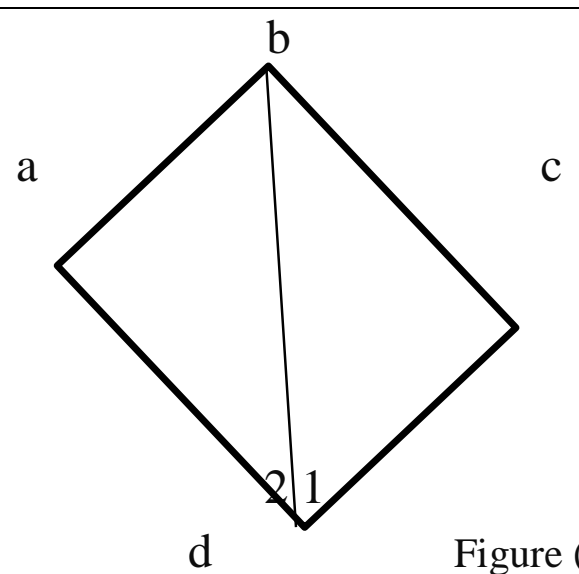

Figure (6.3)

Proof

$$
\begin{aligned}
& { }_{0}^{0} \text { Area of } \quad \text { a b c d }=\frac{(b d)^{2} \times \sin (1) \sin (2)}{\sin (1+2)} \\
& { }_{0}^{0} 0^{0} \mathrm{~m}(1)=\mathrm{m}(2)=\mathrm{m}\left(\frac{d}{2}\right) \\
& \quad \text { from }(1),(2)
\end{aligned}
$$

new method

$$
{ }_{0}^{0}{ }_{0} \text { Area of } \square \text { a b c d }=\frac{(b d)^{2} \times \sin ^{2}\left(\frac{d}{2}\right)}{\sin (d)}
$$

example

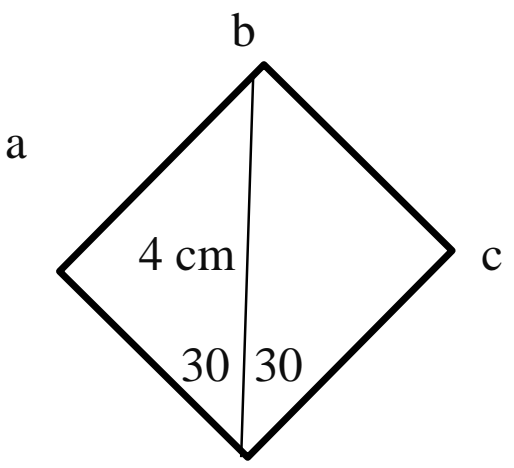

$\mathrm{d}$

Area of $\square$ a b c d $=\frac{(b d)^{2} \times \sin ^{2}\left(\frac{d}{2}\right)}{\sin (d)}$

$$
=\frac{(4)^{2} \times \sin ^{2}(30)}{\sin (60)}=\frac{16 \times \frac{1}{2} \times \frac{1}{2}}{\sqrt{3} / 2}=\frac{8 \sqrt{3}}{3} \mathrm{~cm}^{2}
$$

\section{(2.4)The area of rectangle}




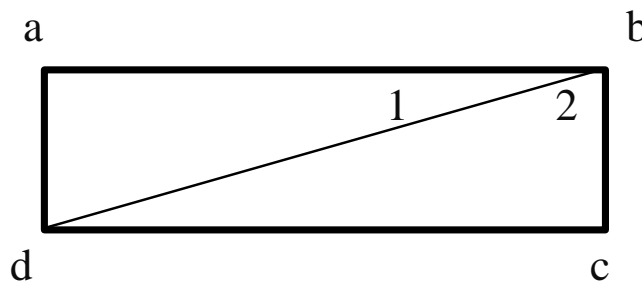

Proof

Figure (6.1)

$\frac{(b d)^{2} \times \sin (1) \sin (2)}{\sin (90)}=(b d)^{2} \times \sin (2) \cos (2)$

Because

$\sin (90)=1$

because $1+2=90$

$\operatorname{Sin}(1)=\cos (2)$

\section{References}


Book calculate areas and volumes of geometric shapes

Carson, P. B. (2012). Effects of levels of formal educational training in mathematics on teacher self-efficacy (Doctoral dissertation, Piedmont College).

Gardner, R. J. (1995). Geometric tomography (Vol. 1). Cambridge: Cambridge University Press

http://www.mathcentre.ac.uk/resources/uploaded/mc-ty-triangleformulae-2009-1.pdf

httpfiles.books.elebda3.netdownload-pdf-ebooks.org-ku-

9199.pdfhttp://www.mathcentre.ac.uk/resources/uploaded/mc-ty-triangleformulae-20091.pdf 